\title{
Diálogos y reconstrucción histórica de la División de Educación Básica
}

\section{Dialogues and Historical Reconstruction of the Department of Basic Education (División de Educación Básica)}

\author{
Cecilia Dobles-Trejos ${ }^{1}$ \\ Universidad Nacional \\ División de Educación Básica del Centro de Investigación y Docencia en Educación \\ Heredia, Costa Rica \\ cecilia.dobles.trejos@una.cr \\ Luz Emilia Flores-Davis ${ }^{2}$ \\ Universidad Nacional \\ División de Educación Básica del Centro de Investigación y Docencia en Educación \\ Heredia, Costa Rica \\ luz.flores.davis@una.cr \\ Pablo Sisfontes-Guilarte \\ Universidad Nacional \\ División de Educación Básica del Centro de Investigación y Docencia en Educación \\ Heredia, Costa Rica \\ pablo.sisfontes.guilarte@una.cr
}

Recibido 3 de junio de 2014 • Corregido 9 de octubre de 2014 • Aceptado 31 de octubre de 2014

\begin{abstract}
${ }^{1}$ Antropóloga social. Profesora e investigadora de la División de Educación Básica del Centro de Investigación y Docencia en Educación (CIDE) de la Universidad Nacional, Costa Rica. Como académica y consultora de organismos nacionales e internacionales se ha desarrollado en los campos de investigación y gestión en educación, investigación y salvaguarda del patrimonio cultural inmaterial, en derechos de poblaciones específicas y gestión de las organizaciones de la sociedad civil.

2 Doctora en Educación, Máster en Educación Superior, catedrática universitaria. Ha ocupado los cargos de directora de Docencia, directora de Desarrollo Profesional y Cooperación Universitaria, presidenta de la Junta de Becas y coordinadora de la Comisión Técnica de Admisión de la Universidad Nacional de Costa Rica. Actualmente, se desempeña como investigadora, extensionista y docente en la División de Educación Básica del CIDE.

${ }^{3}$ Educador graduado de la Universidad Nacional (UNA), Costa Rica. Doctor en Educación de la Universidad La Salle, Costa Rica. Profesor e investigador. Actualmente es director de la División de Educación Básica del Centro de Investigación y Docencia en Educación (CIDE) de la Universidad Nacional, Costa Rica.
\end{abstract}


doi: http://dx.doi.org/10.15359/ree.19-1.19

URL: http://www.una.ac.cr/educare

CORREO: educare@una.cr

Resumen. El objetivo de este artículo es contribuir a la construcción histórica de la unidad académica formadora de maestros y maestras de la Universidad Nacional de Costa Rica (UNA). Con muchas ideas y sueños, en 1983 un grupo académico sienta las bases de lo que hoy conocemos como la División de Educación Básica (DEB) del Centro de Investigación y Docencia en Educación (CIDE). La DEB hereda de la Escuela Normal esa capacidad de reflexión y propuesta de quienes dieron los primeros pasos en la formación de los maestros y maestras del país. Es así como, con muy buenas ideas, se inicia un proceso constante de revisión, reflexión y propuesta, que aún hoy forma parte del quehacer diario de esta unidad académica. A lo largo de su historia, esta visión reflexiva le ha permitido ir planteándose, como "norte", la búsqueda de propuestas novedosas para aportar a la solución de las necesidades educativas del país. El presente artículo hace un recorrido por la historia de esta unidad académica al cumplir su treinta aniversario, enfatizando en los cambios sociales y económicos que a nivel nacional se estaban dando en la década de los años ochenta del Siglo XX y cómo estas coyunturas inciden en la dirección de este quehacer en la actualidad y fundamenta la orientación futura hacia una búsqueda de diversificación de su oferta académica y participación en el desarrollo del país y la región centroamericana. Todo ello, acorde con las demandas sociales y económicas propias del Siglo $\mathrm{XXI}$, tales como un equilibrio entre lo áulico y el contexto social, la influencia de las herramientas tecnológicas y el advenimiento de la virtualidad en la educación, con la consecuente expansión de su influencia pedagógica social en el tiempo y el espacio actual del hecho educativo. Este hecho se caracteriza, hoy, por una visión global y planetaria de la educación que conlleva nuevos retos alrededor de la cooperación internacional, el dominio de varios idiomas y la posibilidad de extender la influencia del quehacer de la DEB allende fronteras. La metodología utilizada en la realización del presente artículo utilizó la triangulación de evidencias obtenidas de la realización de entrevistas con las directoras de la $\mathrm{DEB}$, un taller de sensibilización y reflexión para recuperación de información con el actual equipo académico de la DEB y el análisis documental que incluyó el estudio de reglamentos, planes de estudio y otros que son parte del acervo institucional.

Palabras claves. Formación en educación, pedagogía, educación superior, educación básica, investigación histórica.

Abstract. The present article is aimed at contributing to the historical development of the academic unit in charge of training teachers at Universidad Nacional de Costa Rica (UNA). In 1983 a group of educators with many dreams and ideas built the bases of what we know today as the Department of Basic Education (División de Educación Básica-DEB), part of the Center for Research and Teaching in Education (Centro de Investigación y Docencia en Educación-CIDE) at Universidad Nacional de Costa Rica. The DEB inherited the capacity to reflect and make proposals from the Escuela Normal, the institution that took the first steps in training teachers in the country. In this way, an ongoing process of revision, reflection, and proposals was started full of good ideas and continues to form part of the daily labors of this academic unit. Throughout the history of the DEB, its reflective vision has allowed it to establish its main purpose: searching for innovative proposals to fill the country's educational needs. As part of the $30^{\text {th }}$ anniversary, this article is an overview of the history of this academic unit, paying special attention to the social and economic changes occurring during the 1980 's in the country, and how they influenced the current direction of the unit and how it defines its future orientation towards a diversified academic offering and participation in the development of the country as well as the Central American region. The foregoing is to be established according 
to the social and economic demands that characterize the XXI century, such as a balance between the classroom and the social context, the influence of technology and the introduction of virtual education, and the consequent expansion of its social pedagogical influence in the current time and space. This fact is characterized by an educational global and planetary vision that brings new challenges concerning international cooperation, the command of several languages and the chance to extend DEB's influence beyond its current limits. The methodology used in this article was the triangulation of evidence obtained from interviews with DEB directors, a sensitization and reflection workshop developed with DEB's current academic team in order to recover information, as well as the analysis of regulations, curricula and other institutional documents.

Keywords. Training on education, pedagogy, higher education, basic education, historical research.

La División de Educación Básica, DEB, cumplió en el año 2013 su 30 aniversario vivificándose como una unidad académica que hereda y continúa la labor de formación deeducadoras y educadores emprendida por la Escuela Normal de Costa Rica, y sobre la cual se edifica la Universidad Nacional. "División" al tiempo que "unidad", al unísono, en constante y dinámica interacción. Estos calificativos muy bien representan esta organización viva, caracterizada en la actualidad por la valoración de la diversidad. Esta cualidad dialógica es la que propició que en la elaboración del presente artículo se recurriera a un proceso metodológico participativo en el que se entrevistó a las directoras que ha tenido la DEB en estos 30 años de existencia, se realizó un taller con el personal académico para la reconstrucción histórica y todo la información obtenida de ambos procesos se contrastó con el análisis de documentación y reglamentos de este periodo.

Los grandes desafíos del nuevo milenio, que tienen que ver incluso con la subsistencia de la vida en el Planeta, así como los retos para la construcción de una sociedad más equitativa, solidaria, creativa y comprometida con la vida, implican la creación de alternativas inéditas en la educación. De esta manera, la responsabilidad de participar en la búsqueda de opciones coherentes con los principios de la Universidad Nacional ha sido y continúa siendo el horizonte que orienta la labor que lleva a cabo la División de Educación Básica.

Para dar respuestas a esos desafíos, y en concordancia con lo establecido en el preámbulo del Estatuto Orgánico de la Universidad Nacional (UNA, 1993), la DEB desarrolla programas y proyectos en las diversas áreas académicas: docencia, investigación, extensión y producción.

En la actualidad, esta unidad académica forma docentes en las siguientes carreras de grado:

- Pedagogía con énfasis en I y II Ciclos

- Pedagogía con énfasis en Educación Preescolar

- Educación Especial con énfasis en Integración

- Enseñanza del Inglés en I y II Ciclos 
doi: http://dx.doi.org/10.15359/ree.19-1.19

URL: http://www.una.ac.cr/educare

CORREO: educare@una.cr

Además, a nivel de posgrado ofrece la Maestría en Pedagogía con énfasis en Diversidad en los Procesos Educativos y Maestría en Pedagogía con énfasis en Desarrollo y Atención Integral de la Primera Infancia.

De las carreras indicadas, tres: Pedagogía con énfasis en l y ll Ciclos, Pedagogía con énfasis en Preescolar, y Educación Especial con énfasis en Integración se encuentran re-acreditadas ante el Sistema Nacional de Acreditación de la Educación Superior (SINAES), lo que significa que cumplen con altos estándares de calidad. Es importante destacar que estas fueron las primeras carreras acreditadas en la Universidad Nacional y en el país en el área de educación, responsabilidad asumida por la DEB en concordancia con la herencia de la Escuela Normal, tal como lo indicó, en su momento, la exdirectora de la DEB máster Ana Lorena Vargas Víquez.

La DEB dispone de planes de estudio para cada una de las carreras mencionadas, los cuales comparten una estructura en común conformada por áreas disciplinarias, ejes curriculares, temas transversales, principios metodológicos, principios de evaluación, además de cursos del componente común pedagógico y oferta de cursos optativos.

El estudiantado de la DEB está constituido, en el 2013, por 785 aprendientes provenientes de todo el país.

El personal académico y administrativo se caracteriza por su idoneidad profesional y compromiso con los principios de la Universidad Nacional.

La División de Educación Básica persiste en la búsqueda constante de su mejoramiento para ofrecer a la comunidad nacional y regional nuevas miradas que propicien una educación forjadora de personas y sociedades felices, críticas, creativas y con un decidido compromiso social y ecológico. Por lo anterior, considerando que la educación es un fenómeno social y, por consiguiente, un proceso de transformación de vida en la convivencia, la DEB desarrolla una praxis pedagógica caracterizada por procesos de profunda reflexión en su autoevaluación permanente con fines de mejoramiento, integración de las áreas académicas e incorporación de las nuevas tecnologías en todo el quehacer académico.

\section{El campo de cultivo}

El Pbro. Benjamín Núñez Vargas, primer rector de la Universidad Nacional, expresa lo siguiente (Núñez, 1974, p. 26):

La universidad es más que un simple agregado físico de edificios y personas, de funciones y estructuras burocráticas. Constituye una comunidad auténtica, orgánica y dinámica, en la que todos sus miembros participan de un conjunto vivo de ideales comunes que se encuentran en constante proceso de actualización. 
Y es así como ha cobrado vida la División de Educación Básica a lo largo de los años.

La Escuela Normal de Costa Rica, desde el año 1914 forma educadoras y educadores para la educación primaria hasta 1973, año en que nace la Universidad Nacional y se crea la Escuela de Educación perteneciente a la Facultad de Filosofía, Artes y Letras, en espera de la creación del Centro de Investigación y Docencia en Educación CIDE, según el transitorio al artículo 60 del Estatuto Orgánico de 1976 (Universidad Nacional, 1976).

Diez años después de haber sido inaugurada la Universidad Nacional, en 1983 se conforma el Centro de Investigación y Docencia en Educación (CIDE), en ese entonces don Óscar Benavides Montero es el decano la Escuela de Educación y pasa a ser el primero del CIDE (1983 a 1990).

El Centro creció y se fortaleció con nuevos profesionales y con nuevas ideas para mejorar el panorama educativo del país; en esta línea, para el año 1986 se inicia un procesow de reestructuración del CIDE. Al respecto, plantea Benavides (2008, pp. 155-156) que:

Gracias a la participación de excelentes profesionales, se constituyó un equipo que impulsó el Modelo Organizativo para el Desarrollo Académico del Centro de Investigación y Docencia en Educación (1986). De su contenido-el cual fortaleció y reorientó las acciones del Centro- tomó conceptos que marcan el cometido del CIDE. Se enfatiza que el centro gravitacional de la Institución es el desarrollo académico, y se destaca la vocación para formar recursos humanos para los diversos niveles y modalidades de

La gestión como directora de la señora Lilliana Quesada Yanarella inicia en 1985. En ese primer momento es llamada por el entonces director de la Escuela de Educación de la UNA don Oscar Benavides. Ella asume la dirección de Formación Inicial y le corresponde guiar el proceso de transición para constituir la División de Educación Básica. En 1987 se realiza un proceso de elección formal para la dirección de la DEB y se elige a doña Lilliana en este puesto. Ella estuvo en este cargo hasta 1988, año en que se traslada a trabajar en la Vicerrectoría de Vida Estudiantil.

la educación, así como para la producción científica, tecnológica y humanista en educación.

Con esta reestructuración no solo se definieron las líneas de trabajo futuro, sino que también se sientan las bases para constituir las unidades académicas conocidas hoy. No obstante, es hasta el año 1993, con la aprobación del segundo Estatuto Orgánico de la Institución (Universidad Nacional, 1993), específicamente mediante el artículo 75, que las divisiones adquieren el rango de Unidad Académica.

En los inicios de la conformación del CIDE, su estructura organizativa se sustentaba en distintas áreas de trabajo; entre ellas: formación inicial, formación en servicio, núcleo común, entre otras, que fueron la base de las unidades académicas y carreras que hoy conocemos en el CIDE. 
doi: http://dx.doi.org/10.15359/ree.19-1.19

URL: http://www.una.ac.cr/educare

CORREO: educare@una.cr

Siendo aún director de la Escuela de Educación don Óscar Benavides Montero, se le solicita a la señora Lilliana Quesada Yanarella que asuma la dirección de la en ese entonces llamada "Formación inicial", que es lo que luego de la reestructuración del CIDE se pasó a denominar División de Educación Básica.

La señora Lilliana Quesada Yanarella participa en la reestructuración del CIDE y coordina las primeras acciones que contribuyen a la constitución de la División de Educación Básica, su gestión se extendió desde 1985 hasta 1988. Ella vivió las reuniones, discusiones y estudios que forman parte necesaria de ese proceso de reestructuración del CIDE y, en particular, el paso de transición entre Formación Inicial y la División de Educación Básica (ver figura 1).

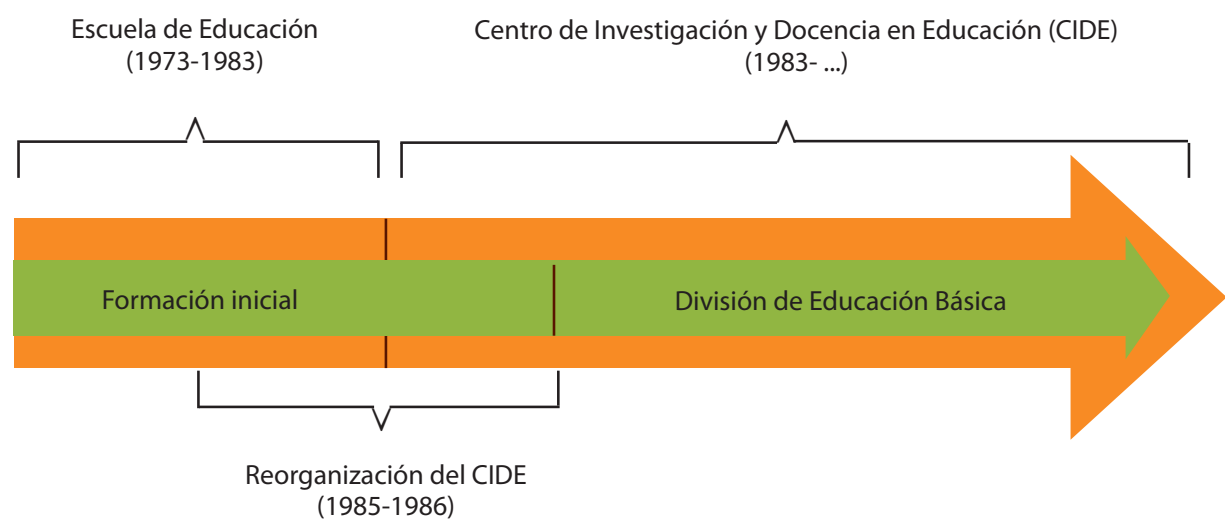

Figura 1. Origen de la División de Educación Básica.

Formación Inicial tenía en ese entonces seis carreras: Artes Industriales, Educación para el Hogar, Orientación, Preescolar, I y II Ciclo, y Problemas de Aprendizaje. Con la reestructuración las carreras de Educación para el Hogar, Orientación y Artes Industriales pasan a formar parte de lo que es hoy la División de Educación para el Trabajo. Las carreras de Preescolar y de I y II Ciclos permanecen en la División de Educación Básica. El plan de estudio de la carrera de Problemas de Aprendizaje se declara terminal y en su lugar se abre la carrera de Educación Especial, dentro de la DEB, según lo expresado por la máster Enriqueta Zúñiga Chaves (E. Zúñiga, comunicación personal, 20 de marzo de 2013), pionera de esa carrera.

\section{Pasos firmes en la consolidación de la Unidad Académica}

Las conversaciones y diálogos de café caracterizaron el quehacer de los ambientes universitarios en el país durante los años 80. Década difícil para Centroamérica, pues la sombra 
de la guerra dibujaba tensiones entre las distintas posiciones políticas; de ahí que alrededor de una taza de café, se podían dirimir tardes enteras cuáles podían ser los mejores caminos para alcanzar los sueños para el futuro del país.

Tardes de café está en las simientes de la DEB, con discusiones académicas en las que se compartieron miedos, dudas, conceptos, visiones de futuro. Según cuentan las docentes que vivieron los inicios de la DEB, a estas tardes de café se llevaban situaciones que se vivían en las aulas universitarias, aciertos o angustias sobre la práctica pedagógica que andaba buscando un punto para poder anclar los sueños. Estas conversaciones muchas veces apasionadas llevaban a discusiones lo suficientemente fuertes para realizar los primeros modelos en la masa de arcilla que se convertiría posteriormente en la División que hoy conocemos.

No podemos dejar de lado lo que pasaba en el país para la década de 1980: crisis del estado benefactor-empresario, crisis financiera, guerra en Centroamérica, cambio en las lógicas productivas, entre otros. En la educación, además, podíamos ver claramente que el país tenía ya cinco universidades privadas y cuatro públicas. Las universidades privadas tendrán un crecimiento exponencial en las dos décadas siguientes hasta llegar a más de 50 en la actualidad. Sin duda, todas estas situaciones definen claramente un contexto que se manifiesta en el quehacer de las academias, pues se buscan nuevas propuestas que contribuyan a estabilizar la situación nacional y poder enrumbar al país.

Viviendo el proceso de reestructuración del CIDE y consolidación de las nuevas unidades académicas, a nivel nacional surge una gran oportunidad para afianzar los ideales que conforman la División de Educación Básica que hoy conocemos. El Ministerio de Educación hace una valoración a nivel nacional y detecta no solo un faltante de docentes de secundaria, sino que también un porcentaje muy alto de maestros y maestras, que no tenían la formación mínima requerida para ejercer, pues trabajaban sin título. De ahí, que propone una alianza con las escuelas de educación de las universidades públicas para resolver ese faltante. A esta propuesta se le llama "Plan de emergencia".

Este plan de emergencia se convierte en una plataforma de acción, que va a ser estratégica para la consolidación de la División de Educación Básica. Pues el Plan de Emergencia se da en paralelo al proceso de reestructuración del CIDE, justo al momento en que empiezan a afianzarse las nuevas unidades académicas. El Plan permite afirmar la visión que se tenía sobre el perfil del profesional y, a su vez, la articulación con la realidad educativa

El plan de emergencia fue algo muy bueno, los estudiantes que me he topado dicen"gracias a la Universidad Nacional, como nos preparó", se dio una formación muy buena. Cuenta en eso el equipo de personas que vinieron a trabajar aquí. Ese plan sí dio resultados positivos.

Lilliana Quesada Yanarella (Directora DEB 19851988). (L. Quesada, comunicación personal, 28 de febrero de 2013) nacional, a la cual se le iba a responder desde una propuesta reflexiva y constructiva. 
doi: http://dx.doi.org/10.15359/ree.19-1.19

URL: http://www.una.ac.cr/educare

CORREO: educare@una.cr

En el estudio realizado por Vargas (2008, p. 84) se plantea que:

... las acciones que hasta ese momento realizaron el MEP y las universidades para enfrentar el problema de la calidad de la formación de educadores, resultaron insuficientes ante el déficit creciente de educadores y la cantidad de aspirantes que laboran sin título, sobre todo en las zonas rurales.

En 1988, el MEP firma un convenio con las universidades estatales para la creación de los Planes de Emergencia. Con estos planes se pretendía cubrir el déficit de maestros y mejorar la formación de los docentes que trabajaban sin poseer título.

Por otro lado, es importante aclarar sobre las situaciones que estaban en los cimientos de la propuesta del Plan de Emergencia; al respecto, Vargas (2008, p. 84), refiriéndose a un estudio realizado en 1993 por Sequeira, A., en torno a los planes de emergencia, revela que:

... la carencia de docentes en primaria se debía, entre otros factores, al crecimiento demográfico, las desfavorables condiciones salariales y la jubilación de los educadores. Era evidente que la carrera de Educación Primaria resultaba poco atractiva por los bajos salarios que se pagaban en ese momento.

Se plantean grandes retos, pues se requerían más profesoras y profesores universitarios dispuestos a subsanar este faltante de educadoras y educadores a nivel nacional. Al calor de estos cambios que consolidan el nacimiento de la DEB se realizan modificaciones en los planes de estudio de las tres carreras. Particularmente, en el caso de la carrera de I y II Ciclo se llevan a cabo ajustes para responder al plan de emergencia.

Antes y durante el proceso de reestructuración del CIDE, las instancias de Formación Inicial y Formación en Servicio trabajan muy unidas. Durante el Plan de Emergencia, compartían un plan de estudios, pero con diferentes modalidades de entrega de la docencia y con poblaciones meta diferentes. Formación Inicial se encargaba de solventar la formación de docentes nuevos y Formación en Servicio de quienes estaban en ejercicio, es decir, que trabajaban pero aún no tenían un título que los acreditara para ello. Esta última modalidad se impartía los fines de semana y viernes en la noche. Formación en Servicio es lo que es hoy la División de Educación Rural. Según recuerda la académica Luz Emilia Flores Davis (comunicación personal, 3 de marzo de 2013), en esa época se coordinaba más entre el profesorado de ambas instancias, e inclusive el personal docente, en algunos casos, daban cursos en la otra modalidad. 
Los estudiantes que participaron en el plan de emergencia, luego se los topaba uno en la calle y me decían "gracias a la UNA, por como nos formó", se dio una formación muy buena. Hubo toda una visión sobre lo que debe ser un educador, que se le logró transmitir a esa generación. (L. Quesada, directora DEB 1985-1988, comunicación personal, 28 de febrero de 2013)

Dentro de la definición curricular de la DEB, el Plan de Emergencia implicó muchos cambios, por ejemplo: disminuía la cantidad de las materias; se enfatizaba en metodologías; los cursos eran interdisciplinarios, sin embargo, una sola persona se tenía que preparar en teoría educativa, psicología, sociología, entre otros, para dar un curso de tres horas. Las personas especialistas se contrataban para nutrir las programas de cursos, no obstante, estas mismas personas debían prepararse para impartir cursos que comprendían diversas áreas del conocimiento, como se mencionó anteriormente, de ahí que para ese momento fuera tan importante la labor de equipo.

Con Lilliana Quesada a la cabeza se trazan las primeras líneas de acción durante la reestructuración, como, por ejemplo, la contratación de profesionales con solvencia y liderazgo en el área de la educación. Según cuenta ella "la clave era la calidad del personal que se logró contratar: Lorena Vargas, Luz Emilia Flores, Olga Solano, Marta Sánchez, Aida Gamboa, se formó un equipo comprometido".

En esa coyuntura se identificaban los grupos de profesionales que habían mostrado idoneidad en el campo pedagógico, luego se entrevistaba a personas y se seleccionaban. Afirma L. Quesada (comunicación personal, 28 de febrero de 2013) que: "Una cosa es sembrar chayotes en la pizarra que sembrarlos en la huerta, que tuvieran una vivencia en el campo". En la entrevista las futuras académicas y académicos debían comprobar que

¡Cómo trabajamos nosotros! la sensación que tengo es que era un trabajo arduo, de mística, el sueño era una mejor educación, formar verdaderos educadores en todas las áreas que nos correspondían. A mí como la Escuela Normal me marcó tanto, formar gente que verdaderamente fuera a formar a otros.

(L. Quesada, comunicación personal, 28 de febrero de 2013) tenían experiencia en las aulas escolares. Así inició el compromiso de las pioneras y los pioneros que trabajaron en la División de Educación Básica.

En la transición de Formación Inicial a la Divísión de Educación Básica, Lilliana Quesada siguió con el cargo de directora; cuando entraron los nuevos reglamentos, se realiza un primer proceso de elección en el año 1988, en el cual ella es reelecta. Sin embargo, poco tiempo después fue nombrada como Vicerrectora de Vida Estudiantil.

En la dirección la sucedió Ana Lorena Vargas Víquez, quien fue electa por primera vez en 1989. La máster Vargas, tanto desde la coordinación de I y II Ciclos, como desde la dirección de la DEB, le dio un gran impulso al Plan de Emergencia. 
doi: http://dx.doi.org/10.15359/ree.19-1.19

URL: http://www.una.ac.cr/educare

CORREO: educare@una.cr

El Plan de Emergencia plantó firmemente los cimientos de la DEB en la búsqueda de una educación de calidad y con un corazón de contribuir a mejorar la propuesta educativa nacional. Sin embargo, aún faltaban ingredientes para consolidar las inquietudes académicas que se conjugaban con las vivencias que, como país, teníamos al final de la década de los 80 . Los procesos de guerra y de firmas de acuerdos de paz, traían nuevos retos a la población costarricense, ahora ya no era mirar hacia los otros países centroamericanos, ahora tocaba voltear la mirada hacia adentro. La crisis de los años 80 deja muchas secuelas en la realidad costarricense.

Ana Lorena Vargas Víquez, con especialidad en currículo, es invitada por la señora Lilliana Quesada a trabajar en el proceso de reestructuración en 1986 y se le ofrece la coordinación de la carrera de I y II Ciclos. La gestión como directora de la señora Vargas abarca el período de 1989 a 1992 y es reelegida nuevamente de 1992 a 1995.

Es cuando encontramos esas grandes carencias que durante una década habían quedado postergadas y brotando por todos los rincones del país, demandando con mucha fuerza tierra, trabajo y una educación de calidad.

Para aquellos académicos y académicas que vivieron la época de 1989 a 1995 en la DEB, ha quedado como una honda huella el trabajo que se realizó durante 6 años en la comunidad de Guararí, en la provincia de Heredia. "Toda la División se movilizó a trabajar en esta comunidad: prácticas, proyectos, cursos de formación continua, etc." (L. Vargas, comunicación personal, 12 de marzo de 2013).

Para esos años se "planta" en la División un liderazgo fuerte, que demanda la equidad de género, capaz de sostener sueños ante viento y marea, para consolidar una visión que hoy se puede leer en los planes de estudio de la DEB, a través de propuestas teórico-epistemológicas sustentadas en la pedagogía crítica. Como sostiene el académico historiador de la DEB Rafael Esteban Jiménez, en el período de dirección de Lorena Vargas hubo una fuerte tendencia a fortalecer la carrera de l y ll Ciclos (Rafael Jiménez, comunicación personal, 01 de agosto de 2012).

Para la época de 1988 a 1996, la propuesta no era filosofar sobre la realidad sino, más bien, poder concretar esas nuevas tendencias de hacer pedagogía en las realidades de las comunidades costarricenses. En este caso particular se afinca el trabajo mediante el proyecto "Calidad de Vida" en la comunidad de Guararí.

He sido muy intuitiva, pragmática y rebelde"

"A mí no me enseñaron a practicar la obediencia debida, las personas valen por lo que hacen y no por lo que son.

(L. Vargas, comunicación personal, 12 de marzo de 2013).

En esa década, las discusiones académicas eran constantes. Quizás en ese momento, como mencionan algunas académicas y algunos académicos que vivieron esos momentos "esa época fue muy dura"; sin embargo, viéndola ahora, casi veinte años después, reconocen que fue un proceso que permitió decantar y pulir el sueño que cada una y uno tenía, sobre todo 
en aquellas y aquellos que iniciaron su participación en este proceso siendo estudiantes de la División y dieron sus primeros pasos como académicos o académicas.

En ese sentido, hoy podemos reconocer que la diversidad de criterios, sueños y visiones que conforman la División de Educación Básica son parte de su identidad, situación que hace recordar las palabras de la máster Lorena Vargas cuando afirma que: "dentro de un proyecto educativo lo básico son los recursos humanos, la formación. Para mí la formación es la independencia, formar personas independientes, por eso el equipo se constituyó no necesariamente por la coincidencia de pensamiento, sino por calidad profesional" (L. Vargas, comunicación personal, 12 de marzo de 2013).

Para 1989 la División de Educación Básica está dando sus primeros pasos. Una de las tareas iniciales para alcanzar la consolidación era la contratación de nuevos y nuevas profesionales, la condición que marcaba

Si queríamos estudiantes críticos, había que posicionarse fuerte y buscar académicos que quisieran crecer con la propuesta. Que fueran capacitables.

Lorena Vargas, Directora 1989-1995 (L. Vargas, comunicación personal, 12 de marzo de 2013) claramente en ese entonces la directora, era que las personas fueran "capacitables", es decir, que tuvieran el interés de crecer académicamente. Es desde esta política que se contó con la participación de académicas y académicos de otras latitudes como Gimeno Sacristán, Saville Kushner, Montserrat Moreno, Rafael Porlán, entre otros. Se organizaron talleres de capacitación que en algunos casos duraban un mes, pero que permitieron acercar al personal académico de la DEB a lo más actualizado en torno a la pedagogía.

El proyecto"Calidad de Vida" ejecutado en Guararí, se sustentaba en la premisa de que las futuras profesionales y los futuros profesionales en educación deben aprender a cuestionar la realidad y en ese proceso determinar cuál es su papel como docentes. Apunta L. Vargas (comunicación personal, 12 de marzo de 2013) que "queríamos que los estudiantes fueran de la práctica a la teoría". Según las académicas y los académicos que acompañaron esta propuesta del 89 al 95, lo que se buscaba era formar personas independientes, conscientes y críticas de la realidad social y económica del país.

El proyecto no solo tenía un objetivo académico, sino que buscaba además impactar positivamente en la realidad educativa de Guararí; por esta razón, con la comunidad se daba una suerte de reciprocidad, pues mientras el estudiantado de la DEB hacía sus prácticas en la escuela, las docentes de las escuelas venían a la universidad para recibir talleres para actualizarse.

En esa época, había buenos elementos en las carreras de la DEB. No todos pensábamos lo mismo, teníamos posiciones muy distintas. Sin embargo, todos nos comprometimos a llevar a cabo esta propuesta de la División.

Lorena Vargas, Directora 1989-1995 (L. Vargas, comunicación personal, 12 de marzo de 2013)

Lo que sucedía en la comunidad de Guararí y en 
doi: http://dx.doi.org/10.15359/ree.19-1.19

URL: http://www.una.ac.cr/educare

CORREO: educare@una.cr

las aulas universitarias era tema para las tardes de café entre el personal académico de la DEB: en torno a una mesa se generaba un espacio de retroalimentación, que se repetía semana a semana. En esas jornadas de discusión, cada quien podía llevar sus inquietudes sobre lo que le servía o no en el aula universitaria, razón por la cual las discusiones podían tornarse fuertes, pero no por eso dejaban de ser constructivas.

\section{Fortaleciendo la estructura}

De 1995 al 2000 asume la dirección Zulay Pereira Pérez, este es un período que plantea nuevos retos para la División, pues como se apuntaba páginas atrás, las direcciones articularon su gestión a un proyecto que fortalecía el programa de gestión durante la administración.

No se puede dejar de lado que en la década de los noventa se vivió una crisis muy seria para las universidades: los gobiernos de turno apostaron a una nueva lógica financiera para subsanar la crisis de los años 80; sin embargo, esta nueva propuesta de administrar nuestras finanzas como país, nos ponía de cara a dar cuentas a los grandes acreedores: Fondo Monetario Internacional y el Banco Mundial. Inician, bajo su tutela, los programas de ajuste estructural, que entre otras cosas, buscan la manera de limitar y controlar el financiamiento

"Yo era muy legalista, me preocupaba porque la gente iba a estar incómoda, pero seguía lo que la normativa establecía". Zulay Pereira (Directora 1995-2000) para las universidades públicas. Fue una década de marchas y protestas por parte de las universidades públicas para demandar lo que era justo, para que dentro de un marco de autonomía pudieran crecer la investigación y la libre cátedra, tan necesarias para fortalecer la democracia.

La presión dentro de la Universidad Nacional también era una realidad, pues se buscaba, con un presupuesto institucional muy limitado, fortalecer la docencia. Desde la División de Educación Básica, esta situación se vivió como una lucha permanente por hacer prevalecer los tiempos asignados a investigación y, a su vez, en la búsqueda estratégica de lograr aumentarlos, pues como apunta Zulay Pereira (Comunicación personal, 8 de abril del 2013):

Fue necesario pararse en seco, para que no nos redujeran los tiempos de investigación. Fue cuando, por ejemplo, para un período se abrieron dos grupos de carrera y no teníamos tiempos para cubrir las necesidades del nuevo grupo. En la Rectoría se nos dijo que no tenían tiempos, que usáramos lo que teníamos para investigación; sin embargo, dijimos que esto no era negociable. Se les dijo a los estudiantes que si querían tener lecciones deberían ir a exigir sus derechos, y así fue, se fueron a la Rectoría y exigieron el nombramiento de sus profesores. En cosa de horas tuvimos los tiempos para poder atender al grupo de estudiantes. 
La División vivió una época en la que se fortalece administrativamente, como lo dice quien dirigió la unidad académica para ese quinquenio, "Soy una persona sumamente ordenada y era necesario para poder fortalecer la División" (Z. Pereira, comunicación personal, 8 de abril del 2013). Esta forma de administrar, aun bajo las condiciones de crisis que se vivían, permitieron fortalecer la unidad académica.

A partir del 2000 asume la Dirección de la DEB Irma Zúñiga León, quien busca posesionar la carrera de Preescolar y Educación Especial no solo en el ámbito la DEB sino también en el CIDE, en razón de la priorización brindada a la carrera de l y ll Ciclos y de las nuevas tendencias y necesidades formativas del acontecer educativo nacional e internacional. Entre sus metas figuraba la creación de opciones de posgrado, luego de un fallido intento por aprobar un plan de estudios de Maestría en Didáctica.

Para esos años da sus primeros pasos la Maestría en Pedagogía con Énfasis en Diversidad, más adelante se ampliará más sobre esta primera propuesta de posgrado de la División de Educación Básica. Sin embargo, es importante señalar la coyuntura que vivía el país en ese momento, sobre todo, porque hacia finales de la década de los noventa se consolidan muchas de las luchas por los derechos de las diversidades, particularmente en Costa Rica se aprueba la Ley 7600 Igualdad de oportunidades para personas con discapacidad (Asamblea Legislativa de la República de Costa Rica, 1996) que abre debates importantes sobre los derechos de las personas con discapacidad y, sobre todo, se convierte en un punto de inflexión en las líneas de trabajo que seguía el Ministerio de Educación Pública en torno al trabajo en las aulas escolares, situación que vuelca los ojos a los planes de estudio de las tres carreras de la División y, en particular, a la carrera de Educación Especial, que asume la discusión de la integración de personas con discapacidad a espacios más abiertos dentro del sistema regular.

Por otro lado, en esos años la DEB impulsa el desarrollo de la investigación, de la lectoescritura y de las relaciones interpersonales. El reto, decía I.Zúñiga (comunicación personal, 2 de mayo de 2013), era mantener el nivel que la DEB había alcanzado en años anteriores.

Este momento histórico estuvo impregnado del proceso de autoevaluación con fines de mejoramiento y acreditación que se logró como una evidencia de la calidad sostenida que venía forjando la unidad desde sus inicios. De acuerdo con I. Zúñiga (comunicación personal, 2 de mayo de 2013), todas las gestiones fueron aportando semilla fértil para lograr la acreditación porque cada una de las experiencias académicas generadas en la DEB, las prácticas cotidianas de la gestión administrativa, las luchas, los retos, las tardes de café, las divergencias de posiciones, entre otros aspectos, sentaron las bases de una unidad académica capaz de superarse. 
doi: http://dx.doi.org/10.15359/ree.19-1.19

URL: http://www.una.ac.cr/educare

CORREO: educare@una.cr

Ana Herrera y yo asumimos el reto de la autoevaluación conocedoras de la trayectoria de la unidad, sabiendo que surgirían fortalezas, pero también debilidades que estábamos en capacidad de superar, como parte de ese proceso continuo de búsqueda de la calidad contextualizada en los desafíos cambiantes derivados de las demandas del contexto, el avance de las disciplinas, y los sueños y aspiraciones de la comunidad educativa de la DEB. Se crea la carrera de Inglés para I y II Ciclos, Ilamada la carrera "cincuenta y cincuenta" dado lo novedoso del diseño curricular, elaborado y puesto en práctica de manera interdisciplinaria e interfacultades. Creo que Giselle Miranda fue la primera coordinadora. Rafael Jiménez participó en el proceso. (I. Zúñiga, comunicación personal, 2 de mayo de 2013)

En el 2003, la máster Zúñiga renuncia a la dirección de la DEB para asumir la decanatura del CIDE, por lo cual asume la dirección Ana Herrera Castro, quien se desempeñaba como subdirectora. El reto estaba en profundizar el trabajo desde las diversidades y con estas, así es que durante este período se busca impactar en las comunidades de mayores necesidades, se trabaja particularmente en la zona de Alajuela, también se trabaja en la Escuela Gran Samaria en Guararí. Los recursos para la ejecución de estos proyectos eran muy limitados, por lo que se buscan recursos de donantes fuera de la universidad.

Para esa época las personas que habían acompañado a la DEB desde su inicio, se empiezan a pensionar, así que surge para esa administración el reto de contratar nuevos académicos y académicas. En ese momento particular entran a trabajar en la DEB una nueva generación de académicos y académicas, que poco conocía de la historia pasada, pero que coincidía en el sueño de una mejor calidad de educación. Así lo menciona el académico Armando Matamoros, quien considera que una de las características de la División es el de ser un espacio de oportunidad, que da la posibilidad para quienes tengan interés en aportar y crecer profesionalmente.

En el año 2005 se nombra a Giselle Miranda Cervantes (G. Miranda, comunicación personal, 2 de mayo de 2013) como Directora de la DEB, quien soñaba con establecer vínculos externos entre una DEB con madurez académica y el resto de unidades del CIDE. Es en este período que se abre el segundo énfasis de la Maestría en Pedagogía y se consolida su coordinación. Además se fortalecieron proyectos interdisciplinarios y se reacreditaron las carreras. No obstante, no concluye el período de su nombramiento por lo cual la entonces subdirectora, Érika Vásquez toma la dirección del año 2008 al 2010. La máster É.Vásquez (comunicación personal, 2 de mayo de 2013) tiene un interés especial por humanizar la relación de la administración académica con el estudiantado y fomentar su participación en las decisiones de la DEB por medio de la figura de representantes de grupo. Promueve las pasantías y giras estudiantiles tanto a zonas indígenas de nuestro país, como a diversos países.

Posteriormente, Pablo Sisfontes Guilarte es nombrado como Director de la DEB en el año 2010 por un período de cinco años. Su propuesta de trabajo se fundamenta en consolidar a 
la DEB dando continuidad al programa establecido por la gestión anterior. Él promueve un trabajo en equipo con la subdirectora, quien lidera la marcha académica de la DEB coordinando con las gestoras y los gestores de carrera, el programa integrador de la academia denominado Desarrollo Académico y Gestión Organizativa (DAGO) con la finalidad de actualizar y fortalecer la oferta académica de la División.

En esta gestión se toman decisiones estratégicas orientadas a enfrentar los desafíos del Siglo XXI, tales como la reorganización de los programas, proyectos y actividades que combinan la integración de personal académico experimentado con el más joven. Se orientan dentro de una línea estratégica a establecer relaciones entre la educación y salud, ejes fundamentales en el desarrollo social del país. La intención es constituirse en una unidad académica más competitiva en la búsqueda de posicionarse en una coyuntura que le permita la innovación de la oferta académica para atender las demandas futuras; además de los espacios actuales generados por la oferta curricular, en espacios emergentes y no formales y de gran necesidad de aportar al desarrollo de sectores más necesitados. En este sentido, hoy se promueven acciones innovadoras dentro de la pedagogía social. Asimismo, se pretende abrir espacios para la apropiación de las TIC, con la finalidad de impactar el quehacer académico de la DEB y ampliar su influencia en escenarios educativos aún no accesados por la División en sectores alejados del campus universitario y más allá de las fronteras.

\section{Oferta curricular a nivel de posgrado}

El Pacto Internacional sobre los Derechos Económicos, Sociales y Culturales (1966), firmado en 1976, abre una puerta para cambiar las mirada sobre la otredad; sin embargo, no fue sino hasta la década de los noventa, cuando con más fuerza se incorporan los preceptos básicos que revolucionaron la discusión de los derechos humanos en el mundo y, se empiezan a poner en vigencia en distintos ámbitos de discusión mundial: derechos de los indígenas, derechos de las mujeres, derechos de las personas con discapacidad, derechos de la niñez y quizás sea esta última Convención sobre los Derechos de la Niñez la que pone el dedo en la llaga, cuando establece el interés superior del niño como punta de lanza para hacer valer los derechos de este grupo de población. Cambiar la visión de la niñez como objeto de derechos a ser sujeto de derecho, concreta un anhelo de la humanidad, donde podemos ver que las personas no son objetos del interés del otro, sino que, como apunta Freire sabiamente, debemos darles la posibilidad de constituirse en sujetos durante el acto "creativo" del quehacer pedagógico.

Difícil reto, pues nos da vuelta a la manera de pensar y hacer la pedagogía a la que estamos acostumbrados y que propone nuevas líneas de debate en la División de Educación Básica, ahora los temas son la diversidad, la educación inclusiva, entre otros. Las tardes de café ahora plantean los retos de revisar profundamente y consistentemente con los discursos que 
doi: http://dx.doi.org/10.15359/ree.19-1.19

URL: http://www.una.ac.cr/educare

CORREO: educare@una.cr

demanda el momento, para poder traducirlos en acciones dentro del aula universitaria, en las propuestas de investigación y en el trabajo en las comunidades.

La discusión en torno a la diversidad arranca a inicio del nuevo milenio, y se convierte en la tierra de cultivo para el diseño de la Maestría en Pedagogía, al respecto Jiménez (2007, p. 18), académico de la DEB señala:

Pudiese pensarse que una instancia formadora de mastros y maestras que no posee esos enfrentamientos, esté pasando por un momento de armonía, pero esa armonía puede esconder el quehacer universitario de la reflexión y la confrontación de ideas, lo cual es paralizante y peligroso, para la conformación de un ambiente crítico y propositivo. De los enfrentamientos pedagógicos abiertos y fuertes que vivió la DEB, en los años noventa, la Maestría en Pedagogía recogió los frutos de una batalla académica y conceptual.

La Maestría en Pedagogía es una carrera de posgrado profesional que ofrece la División de Educación Básica. Tiene dos énfasis, a saber: Diversidad en los procesos educativos, que se ofrece desde el año 2002; así como Desarrollo y atención integral de la primera infancia, que inicia en el año 2007. Ambos énfasis comparten un tronco común pedagógico. Este se desarrolla desde la pedagogía constructivista, socio-crítica.

Este proyecto de posgrado, como bien se expresa en sus documentos:

Pretende ser tan vivo como lo es el acto de aprender y se consolida en la búsqueda reflexiva, ética y creativa de experiencias pedagógicas que propicien la formación en la vida y para la vida, tomando la diversidad, integralidad y la complejidad como norma en un enfoque epistemológico dialéctico... . La Maestría en Pedagogía tiende a la búsqueda de una educación sin exclusiones, equitativa, vital, que se sustente en el gozo por la vida en todas sus formas y procure aportar al disfrute de una formación integral; además, funcione como herramienta de emancipación y cambio para los y las actores [sic] involucrados o [involucradas]; en procura del desarrollo de sociedades democráticas, solidarias y ecosostenibles. (Belderbos, s. f., pp. 1-2)

\section{Carreras de grado: praxis pedagógica}

Los planes de estudio de la DEB operacionalizan la visión y misión de la División, del Centro de Investigación y Docencia en Educación (CIDE), y de la Universidad Nacional, para responder a demandas y necesidades de la comunidad nacional, teniendo presente que la dinámica social es cambiante y dinámica. 
Por tres décadas la División de Educación Básica ha formado educadoras y educadores en Educación Preescolar, I y II ciclos y Educación Especial. Actualmente estas carreras se ofrecen a nivel de licenciatura, con grado de bachillerato con salida lateral de diplomado.

Las carreras mencionadas comparten las siguientes áreas disciplinarias: científicapedagógica, de desarrollo humano integral y socio-histórica cultural. Así como los ejes curriculares de investigación, metacognición, práctico-pedagógico y humanístico.

Por su parte, los temas transversales permiten que el estudiantado esté en contacto con la realidad social, estos son: multiculturalidad, género, derechos humanos, ecología y sostenibilidad, poblaciones diferenciadas, educación para la paz y marginalidad.

Los principios metodológicos que se presentan en los planes de estudio son los siguientes: enseñanza constructiva, funcionalidad de los aprendizajes, significatividad de los aprendizajes, interactividad, aprender a aprender, interdisciplinariedad del conocimiento y mediación pedagógica (Universidad Nacional. División de Educación Básica, 2005).

Desde el año 2001, la DEB amplía su oferta académica al incluir la formación de docentes en la Enseñanza del Inglés para I y II ciclos, carrera que se asume conjuntamente con la Escuela de Literatura y Ciencias del Lenguaje en el contexto de una experiencia considerada pionera, por haber logrado el diseño de un plan de estudios con un adecuado equilibrio entre el componente de lengua y el componente pedagógico.

Como se indica en los planes de estudio de las carreras de la División (Universidad Nacional. División de Educación Básica, 2008): a lo largo de la historia de las carreras, se han desarrollado múltiples enfoques epistemológicos, los cuales han intentado ser respuestas oportunas a grandes desafíos pedagógicos, de esa forma las carreras han sentido la influencia de enfoques tales como: normalista, academicista, tecnológico, participativo, pedagogía operatoria, alternativas metodológicas inspiradas en la epistemología constructivista, aporte de tendencias críticas de la pedagogía, entre otros. Estos enfoques han sido asimilados e interpretados desde la perspectiva de un análisis situacional del hecho educativo. Todo ese transitar pedagógico ha permeado el quehacer de las carreras y ha ido generando una madurez teórica-práctica que facilita la formulación de planes de estudio flexibles y abiertos al cambio.

Las carreras han logrado alcanzar un principio orientador y compartido acerca de la naturaleza misma del conocimiento, el cual responde a que el desarrollo académico curricular de la División de Educación Básica se ha promovido a partir de la búsqueda de la ruptura de los modelos lineales de enseñanza, para optar por alternativas que guarden correspondencia con la opción de generar propuestas constructivistas por parte de sus aprendientes. De esa forma, se ha partido del supuesto epistemológico de que no existe conocimiento fuera de la mente del sujeto cognoscente y de que, por lo tanto, una 
doi: http://dx.doi.org/10.15359/ree.19-1.19

URL: http://www.una.ac.cr/educare

CORREO: educare@una.cr

adecuada educación es aquella que propicia experiencias de aprendizaje y que permite, por ello la existencia de condiciones adecuadas para la interiorización y apropiación del conocimiento. Este salto cualitativo ha significado el tener la conciencia de que las respuestas para una educación dentro del marco de esta opción no están agotadas y de que deben generarse grandes esfuerzos académicos para construir respuestas metodológicas adaptadas a las condiciones situacionales de nuestro entorno.

Este sentimiento crítico de ubicación de los planes de formación de la División de Educación Básica ha procurado hacer evidente, en sus actividades académicas, esta vocación de análisis social, generando esfuerzos para formar educadores y educadoras en sus especialidades de cara a la realidad situacional costarricense, y en el contexto de renovadas concepciones educativas. Este reto impone, por un lado, el estar al día con las evaluaciones de la realidad socioeconómica costarricense, de sus necesidades y expectativas y, por otro, generar procesos de investigación que permitan estudiar y experimentar con elementos aportados por la vanguardia de las innovaciones pedagógicas, para proponer nuevos modelos educativos.

De lo anterior, es importante inferir que la formación profesional de nuevas y nuevos graduados no se concibe separada de los esfuerzos de investigación y extensión que realiza la División, como tampoco de los procesos de innovación educativa que se llevan a cabo en las actividades de docencia propiamente. Se concibe, por lo tanto, el desarrollo de la investigación, la docencia, la extensión, y la producción de manera integrada en la realidad concreta y desde ella. De esta manera se cree poder facilitar la realimentación y comprensión de la realidad educativa a la que se debe y se pretende responder con los planes de estudio.

Siguiendo lo anterior, es importante mencionar que la División de Educación Básica ha logrado alcanzar un crecimiento en diferentes áreas del conocimiento vinculadas con la educación, de esa forma se perfila, sobre todo, un desarrollo en cuanto a : La educación como un elemento sociocultural e histórico, la educación como un elemento del desarrollo humano integral, lo cual converge en una posición pedagógica científica, entendiendo la pedagogía como la ciencia que estudia la educación en sus diferentes manifestaciones.

\section{Acreditación de las carreras}

La División de Educación Básica es una de las unidades académicas pioneras en participar en los procesos de acreditación de la educación superior costarricense. La cultura de autoevaluación desarrollada desde sus inicios promovió su participación en las primeras experiencias de acreditación de las carreras de la Universidad Nacional ante el Sistema Nacional de Acreditación de la Educación Superior, SINAES. 
Las carreras de I y II Ciclos, Preescolar y Educación Especial se acreditaron por primera vez en el 2002, luego en el 2007 se reacreditan nuevamente las tres carreras.

Como se establece en el informe final del proyecto "Seguimiento permanente a los planes de mejoramiento derivados de la reacreditación de las carreras de la DEB". Enero 2008-Diciembre 2012 (Ruiz, 2013, p. 23):
La acreditación es un punto muy importante en la historia de la DEB, porque permite un proceso de reflexión, mejora continua y de revisión interna.

(Participante en Taller de reflexión sobre la DEB, 22 de abril de 2013)

El desarrollo de los Planes de Mejoramiento a largo de estos años, ha permitido no solo superar debilidades, sino también establecer nuevos indicadores de logro, que de alguna manera han provocado que las carreras acreditadas desarrollen mayor trabajo en equipo y también identifiquen nuevas oportunidades de mejora, que no necesariamente están dictadas en el informe de mejoramiento.

En el año 2011 es acreditada por tercera vez la carrera de Educación Especial y se entra al proceso de elaboración de informes y revisión de planes de mejora para la reacreditación de las carreras de Preescolar y I y II Ciclo, lo cual se logra a finales del año 2013.

Todo este proceso ha generado debates al interior de la División, los cuales han fructificado en la revisión permanente del quehacer de la DEB, lo que se hace acompañar de nuevas propuestas teóricas, epistemológicas y metodológicas en las áreas de la construcción de saberes pedagógicos. En ese sentido, Ruiz (2013) sostiene que la acreditación ha tomado un protagonismo dentro de la DEB, sobre todo porque se dispone de un mecanismo permanente de sistematización del quehacer de las carreras, lo cual posibilita la reflexión sustentada y la propuesta de cambios, como lo ha sido el caso de la investigación, eje transversal en los planes de estudio.

Los procesos de acreditación implican largas jornadas de trabajo para poder recabar toda la información que forma parte de los requisitos solicitados por SINAES. Todo este proceso es importante para la unidad académica, pues se crea una base informativa necesaria para guiar el apoyo de la gestión administrativa y para el diseño de propuestas en la actualización permanente de las carreras. Además, agrega Ruiz (2013), la acreditación pone nuevamente en la agenda de la DEB a los egresados, empleadores, estudiantes y el contexto de la realidad educativa nacional, la cual es información fundamental para la toma de decisiones en el trabajo administrativo y académico.

\section{De las tardes de café a la era digital}

La División de Educación Básica continúa repensándose y reconceptualizando su quehacer de manera constante, teniendo siempre como desafío el mejoramiento de la 
doi: http://dx.doi.org/10.15359/ree.19-1.19

URL: http://www.una.ac.cr/educare

CORREO: educare@una.cr

educación costarricense. Han variado las estrategias organizativas y se ha pasado del énfasis en las tardes de café, a la comunicación por medios tecnológicos, inspirada siempre en el compromiso ecológico y social.

Cada vez más se ha ido usando la tecnología y sus herramientas en la cotidianidad académica y administrativa en la DEB. En la actualidad, por citar algunos ejemplos, debido a las características propias del nuevo siglo, la DEB ha incorporado la modalidad "presencial y a distancia" en las licenciaturas, lo que ha llevado a las académicas, académicos y estudiantes a incorporar, paulatinamente, innovaciones metodológicas que incluyen las herramientas tecnológicas, tales como el uso de foros virtuales, el correo electrónico, documentos digitales, virtualización de cursos e introducción de cursos en línea en la plataforma "Moodle". Asimismo, la inversión en el uso de equipos que incluye computadores portátiles, "video Beans" y, últimamente, el advenimiento de las pizarras interactivas abren nuevos desafíos con la introducción de la tecnología en la DEB.

Hoy más que nunca, la era digital y de la información nos induce a vincularnos de manera global con la realidad planetaria, lo cual conlleva una serie de desafíos y retos en el desarrollo futuro de la DEB, tales como el bilingüismo, los convenios internacionales con universidades de prestigio internacional y una oferta académica innovadora y actualizada que aporte no solo al desarrollo social y económico del país con una visión ecológica y sostenible, sino de la región centroamericana y del planeta Tierra como un todo.

\section{Referencias}

Asamblea Legislativa de la República de Costa Rica. (29 de mayo de 1996). Ley 7600 Igualdad de oportunidades para las personas con discapacidad. Diario Oficial La Gaceta No. 112. Recuperado de http://www.fonabe.go.cr/Informacion/Reglamento/Documents/Ley\%20 7600\%20Reglamento\%20lgualdad\%20de\%200portunidades.pdf

Belderbos. (s. f.). Maestría Pedagogía. Recuperado de http://www.cide-basica.una.ac.cr/ index.php?view $=$ article $\&$ catid $=47 \% 3$ Amaestria-en-pedagogia\&id $=53 \% 3 \mathrm{Amaestria}-$ pedagogia\&format $=$ pdf\&option $=$ com content $\&$ temid $=142$

Benavides, O. (2008). Los comienzos del CIDE. Revista Electrónica Educare, 12(1), 153-157. Recuperado de http://www.revistas.una.ac.cr/index.php/EDUCARE/article/view/1425

Jiménez, R. (2007). Una necesidad curricular ante los procesos homogeneizantes de la educación contemporánea. Revista Electrónica Educare, 1 (Extraordinario), 11-26. Recperado de http:// www.revistas.una.ac.cr/index.php/EDUCARE/article/view/1353

Núñez, B. (1974). Hacia la universidad necesaria. Heredia, Costa Rica: Editorial Universidad Nacional. 
Revista Electrónica EdUCARE (EdUCARE EleCtronic JOURNAL) EISSN: 1409-4258 VOL. 19(1) ENERO-ABRIL, 2015: 353-373

doi: http://dx.doi.org/10.15359/ree.19-1.19

URL: http://www.una.ac.cr/educare

CORREO: educare@una.cr

Pacto Internacional sobre los Derechos Económicos, Sociales y Culturales (16 de diciembre 1966). Recuperado de http://www.acnur.org/t3/fileadmin/scripts/doc.php?file=biblioteca/ pdf/0014

Ruiz, S. (2013). Seguimiento permanente a los planes de mejoramiento derivados de la reacreditación de las carreras de la DEB. Enero 2008-diciembre 2012. (Informe Final Código 050607). Universidad Nacional. División Educación Básica. Manuscrito inédito.

Universidad Nacional. (1976). Estatuto Orgánico de la Universidad Nacional. Heredia, Costa Rica: EUNA.

Universidad Nacional. (1993). Estatuto Orgánico de la Universidad Nacional. Recuperado de http://www.sia.una.ac.cr/index.php?option=com remository\&ltemid=76\&func=startdo wn\&id $=19$

Universidad Nacional. División de Educación Básica. (2005). Plan de Estudios Licenciatura en Pedagogía con énfasis en I y ll Ciclos de la Educación General Básica. Bachillerato en Pedagogía con énfasis en I y ll Ciclos de la Educación General Básica con salida lateral de Diplomado. Heredia, Costa Rica: Autor.

Universidad Nacional. División de Educación Básica. (2008). Plan de Estudios Licenciatura en Pedagogía con énfasis en ly ll Ciclos de la Educación General Básica. Bachillerato en Pedagogía con énfasis en I y II Ciclos de la Educación General Básica con salida lateral de Diplomado (Reimpresión). Heredia, Costa Rica: Autor.

Vargas, C. A. (2008). Diagnóstico situacional de la labor docente y de la oferta de formación de educadores rurales en Costa Rica. Revista Electrónica Educare, 12 (Extraordinario), 79-97. Recuperado de http://www.revistas.una.ac.cr/index.php/EDUCARE/article/view/1458

\section{Cómo citar este artículo en APA:}

Dobles-Trejos, C., Flores-Davis, L. E. y Sisfontes-Guilarte, P. (enero-abril, 2015). Diálogos y reconstrucción histórica de la División de Educación Básica. Revista Electrónica Educare, 19(1), 353-373. doi: http://dx.doi.org/10.15359/ree.19-1.19

Nota: Para citar este artículo en otros sistemas puede consultar el hipervínculo "Como citar el artículo" en la barra derecha de nuestro sitio web:

http://www.revistas.una.ac.cr/index.php/EDUCARE/index 